\title{
Editorial
}

Ian Farrance and Robert Frenkel

\section{Measurement uncertainty and the importance of correlation}

https://doi.org/10.1515/cclm-2020-1205

Since its introduction in 1993, the Evaluation of measurement data-Guide to the expression of uncertainty in measurement (GUM) [1], has provided the basic framework for evaluating measurement uncertainty (MU). The procedures described in the GUM have been widely applied across all scientific disciplines and increasingly in the medical sciences. However, the recent letter by Rigo-Bonnin and Canalias again highlights the importance of GUM procedures and their contribution to the uncertainty evaluation of derived biological quantities [2].

Most will be familiar with the general concepts of MU as they relate to imprecision and bias (systematic error). Imprecision in clinical laboratory methods is usually evaluated using internal quality control (IQC) procedures, with standard uncertainty $(u)$ obtained from the standard deviation of replicate control results [3-5]. The routine evaluation of bias is usually obtained from proficiency testing surveys when direct traceability studies are impractical or unavailable. The procedures for handling bias and its effects on laboratory results are also the subject of an on-going debate [6-9].

However, not all measurands or quantity values are provided by direct analytical measurement. In some situations, a quantity value is calculated from other measurements through a functional relationship, where the output value (the calculated quantity) is derived from one or more input quantities by applying a defined mathematical equation. The many examples of quantity values which are calculated using this approach have been clearly demonstrated by Rigo-Bonnin and Canalias in their recent letter [2]. Other more 'complex' examples which also provide and explain the relevant statistical principles include: the

Ian Farrance, Discipline of Laboratory Medicine, School of Health and Biomedical Sciences, RMIT University, 3083 Bundoora, Victoria, Australia, E-mail: ian.farrance@rmit.edu.au. https://orcid.org/00000002-2993-0629

Robert Frenkel, Former affiliation: National Measurement Institute, West Lindfield, NSW, Australia amount of albumin excreted in urine [10,11]; the estimated glomerular filtration rate (eGFR) by both the Chronic Kidney Disease Epidemiology Collaboration (CKD-EPI) and the Modification of Diet in Renal Disease Study (MDRD) procedures $[10,12,13]$; the international normalized ratio (INR) $[10,14]$; serum free testosterone calculation by both the Vermeulen and Sartorius procedures [10, 13]; and the DNA methylation ratio [10].

The use of empirically derived functional relationships may vary from relatively simple forms, such as the anion and osmolal gap calculations, to complex forms such as the various eGFR and free testosterone equations. However, the empirical constants which are determined as part of these more complex regression-based equations are often considered to be free of inherent uncertainty, but equations derived by best-fit numerical analysis of patient data will always incorporate a degree of variability as shown by the scattering of raw data points in a " $y$ " vs. " $x$ " scatter graph. Numerical constants derived in this manner must also have associated standard uncertainties. As a consequence, any numerical constants which form part of such calculations are not true constants, but contribute an uncertainty which potentially increases the estimated interval in which the actual quantity value may be found [3, 12]. Unless the standard uncertainties associated with such "constants" are also provided and included in any calculations, the full impact on the output of the functional relationship which is now applied to new input variables cannot be accurately assessed. This assessment is important as it potentially limits the interpretation of a given result when compared to a population reference interval or a fixed clinical decision value, or when assessing the significance of a change in the serial results of a patient (reference change value calculations). In a similar manner to the propagation of measurement uncertainties, the propagation of biological variation has also been described using a procedure derived from the GUM [12].

The data provided by Rigo-Bonnin and Canalias also demonstrates the utility and practical importance of including the correlation term in many of the calculations. Their approach also demonstrates a less obvious consequence of including correlation: that is; the possibility of a 
lower uncertainty for the output quantity by including correlation, when compared to not including or assuming a zero correlation. As briefly shown in the Clinical and Laboratory Standards Institute (CLSI) approved guideline Expression of measurement uncertainty in laboratory medicine [11], a decrease in the uncertainty which propagates to the output quantity may occur and depends jointly on the sign of the correlation coefficient and the sign(s) of the various terms in the GUM propagation equation. The initial example provided by Rigo-Bonnin and Canalias clearly shows this aspect of uncertainty propagation; it describes the calculation of bicarbonate in blood, a derived quantity obtained from the measurements of $\mathrm{pH}$ and $\mathrm{pCO}_{2}$. The important place of correlation (and the Pearson correlation coefficient in particular) in uncertainty calculations should not be underestimated and some additional comments may be useful. If we consider the measurand $z$ as a function of only two inputs, $x$ and $y$, that is:

$$
z=f(x, y)
$$

the standard uncertainties $u(x)$ in $x$ and $u(y)$ in $y$ will propagate into $z$, creating a corresponding standard uncertainty $u(z)$ in $z$, according to the GUM formula [1]:

$$
\begin{aligned}
u^{2}(z)= & \left(\frac{\partial z}{\partial x}\right)^{2} u^{2}(x)+\left(\frac{\partial z}{\partial y}\right)^{2} u^{2}(y) \\
& +2 r(x, y)\left(\frac{\partial z}{\partial x} \frac{\partial z}{\partial y}\right) u(x) u(y)
\end{aligned}
$$

where the correlation (correlation coefficient) between $x$ and $y$ is denoted by $\mathrm{r}(x, y)$. Given a set of $\mathrm{n}$ pairs of quantities $x_{i}$, $y_{i}, i=1,2,3, \ldots, \mathrm{n}$, their correlation coefficient $\mathrm{r}(x, y)$ can be calculated using the following general formula $[1,10]$ :

$$
r(x, y)=\frac{\sum_{i=1}^{n}\left(x_{i}-\bar{x}\right)\left(y_{i}-\bar{y}\right)}{\sqrt{\sum_{i=1}^{n}\left(x_{i}-\bar{x}\right)^{2} \sum_{i=1}^{n}\left(y_{i}-\bar{y}\right)^{2}}}
$$

where $\bar{x}=\frac{1}{n} \sum_{i=1}^{n} x_{i}$ is the mean of the $x$ 's, and $\bar{y}=\frac{1}{n} \sum_{i=1}^{n} y_{i}$ is the mean of the $y$ 's.

Even though the above steps (Eqs. (1)-(3)) have been well described by Rigo-Bonnin and Canalias, and by others previously $[3,10,15]$, it is important to consider these as the 'basic' uncertainty equations. In addition, a key mathematical property of the Pearson correlation coefficient is its invariance with respect to origin or scale in the $x$ 's or $y$ 's. That is; if $x_{i}$ is replaced by $k_{1} x_{i}+k_{2}$ and $y_{i}$ is replaced by $k_{3} y_{i}+k_{4}$, where the $k$ 's are all constants, $\mathrm{r}(x, y)$ remains the same. This invariance holds for all values of $\mathrm{r}(x, y)$ and is essential if any worthwhile meaning is to be attached to a correlation coefficient, which would otherwise depend on such arbitrary matters as the units of measurement and would therefore have little general significance.
The following examples demonstrate some important features of correlation and its impact on the output value in an uncertainty calculation, particularly if any of the input variables do actually show a degree of mutual correlation.

\section{Example 1}

If $z=x+y$, then:

$$
\frac{\partial z}{\partial x}=+1 \text { and } \frac{\partial z}{\partial y}=+1
$$

and Eq. (2) simplifies to:

$$
u^{2}(z)=u^{2}(x)+u^{2}(y)+2 r(x, y) u(x) u(y)
$$

Case 1(a); $r(x, y)=0$, then:

$$
u^{2}(z)=u^{2}(x)+u^{2}(y) \text { and } u(z)=\sqrt{u^{2}(x)+u^{2}(y)}
$$

This is the familiar root-sum-square of two component standard uncertainties, when these describe the uncorrelated combined variation in two input quantities.

Case $1(\mathrm{~b}) ; \mathrm{r}(x, y)=+1$, then:

$$
u^{2}(z)=u^{2}(x)+u^{2}(y)+2 u(x) u(y)=[u(x)+u(y)]^{2}
$$

or:

$$
u(z)=u(x)+u(y)
$$

When the measurand is the sum of the input quantities which have perfect positive correlation, the standard uncertainty of the measurand is the simple linear sum of the standard uncertainties of the two input quantities.

Case $1(\mathrm{c}) ; \mathrm{r}(x, y)=-1$, then:

$$
u^{2}(z)=u^{2}(x)+u^{2}(y)-2 u(x) u(y)=[u(x)-u(y)]^{2}
$$

or:

$$
u(z)=u(x)-u(y)
$$

As always when taking a square root, the result can be either positive or negative. Since an uncertainty cannot be negative, the sign of $u(x)-u(y)$ is so chosen as to make $u(z)=u(x)-u(y)$ positive. That is; $u(z)=|u(x)-u(y)|$. In this situation, it is also possible for $u(z)$ to be zero. That is, with perfect negative correlation $(\mathrm{r}(x, y)=-1)$ it is possible for $u(z)$ to be zero, when $u(x)$ and $u(y)$ are equal and the measurand $z$ is the sum of $x$ and $y(z=x+y)$. This can also occur in other similar situations, depending on the actual numerical values of the terms involved.

\section{Example 2}

If $z=x-y$, then:

$$
\frac{\partial z}{\partial x}=+1 \text { and } \frac{\partial z}{\partial y}=-1
$$


and Eq. (2) simplifies to:

$$
u^{2}(z)=u^{2}(x)+u^{2}(y)-2 r(x, y) u(x) u(y)
$$

Case $2(\mathrm{a}) ; \mathrm{r}(x, y)=0$, then:

$$
u^{2}(z)=u^{2}(x)+u^{2}(y) \text { and } u(z)=\sqrt{u^{2}(x)+u^{2}(y)}
$$

Again, this is the familiar root-sum-square of two component standard uncertainties when they describe the uncorrelated variation in two input quantities. The cases $z=x+y$ and $z=x-y$ are therefore equivalent, as far as the standard uncertainty of $z$ is concerned and as long as $x$ and $y$ are mutually uncorrelated.

Case 2(b); $r(x, y)=+1$, then:

$$
u^{2}(z)=u^{2}(x)+u^{2}(y)-2 u(x) u(y)=[u(x)-u(y)]^{2}
$$

or:

$$
u(z)=u(x)-u(y)
$$

With perfect positive correlation, the standard uncertainty of the measurand in this example is the simple linear difference of the standard uncertainties of the two input quantities.

Again, it is possible for $u(z)$ to be zero when $u(x)=u(y)$ and $z=x-y$ with perfect positive correlation.

Case 2(c); r $(x, y)=-1$, then:

$$
u^{2}(z)=u^{2}(x)+u^{2}(y)+2 r(x, y) u(x) u(y)
$$

or:

$$
u(z)=u(x)+u(y)
$$

In summary, when correlation is associated with the input variables in a measurement uncertainty equation, consideration of its contribution to the final output uncertainty is also required. If $z=x+y$, or $z=x-y$, both the sign of the correlation coefficient and the signs of the input variables jointly determine if the uncertainty associated with the output quantity increases or decreases as a consequence of the correlation. The inclusion of a correlation term in the calculation of uncertainty may actually provide a lower uncertainty value for the measurand or derived quantity value under consideration.

Research funding: None declared.

Author contributions: All authors have accepted responsibility for the entire content of this manuscript and approved its submission.

Guarantor: IF.

Competing interests: Authors state no conflict of interest.

\section{References}

1. Bureau International des Pois et Mesures. JCGM 100:2008, Evaluation of measurement data-guide to the expression of uncertainty in measurement (GUM). Available from: http://www.bipm.org/en/publications/guides/gum.html [Accessed Aug 2020].

2. Rigo-Bonnin R, Canalias F. Measurement uncertainty estimation for derived biological quantities [letter]. Clin Chem Lab Med 2020; 59:e1-e7.

3. Farrance I, Frenkel R. Uncertainty of measurement: a review of the rules for calculating uncertainty components through functional relationships. Clin Biochem Rev 2012;33:49-75.

4. Braga F, Panteghini M. The utility of measurement uncertainty in medical laboratories. Clin Chem Lab Med 2020;58:1407-13.

5. Braga F, Pasqualetti S, Aloisio E, Panteghini M. The internal quality control in the traceability era. Clin Chem Lab Med 2020. https://doi.org/10.1515/cclm-2020-0371 [Epub ahead of print].

6. Magnusson B, Ellison SLR. Treatment of uncorrected measurement bias in uncertainty estimation for chemical measurements. Anal Bioanal Chem 2008;390:201-13.

7. Theodorsson E, Magnusson B, Leito I. Bias in clinical chemistry. Bioanalysis 2014;6:2855-75.

8. Frenkel RB, Farrance I. A statistical procedure for the assessment of bias in analytical methods using conditional probabilities. Accred Qual Assur 2017;22:265-73.

9. Frenkel R, Farrance I, Badrick T. Bias in analytical chemistry: a review of selected procedures for incorporating uncorrected bias into the expanded uncertainty of analytical measurements and a graphical method for evaluating the concordance of reference and test procedures. Clin Chim Acta 2019;495: 129-38.

10. Frenkel RB, Farrance I. Uncertainty in measurement: procedures for determining uncertainty with application to clinical laboratory calculations. Adv Clin Chem 2018;85: 149-211.

11. Clinical and Laboratory Standards Institute (CLSI). Expression of measurement uncertainty in laboratory medicine; approved guideline. Wayne, PA: CLSI; 2012, Document EP29-A, CLSI.

12. Farrance I, Badrick T, Frenkel R. Uncertainty in measurement: a review of the procedures for determining uncertainty in measurement and its use in deriving the biological variation of the estimated glomerular filtration rate. Pract Lab Med 2018;12: e00097.

13. Farrance I, Frenkel R. Uncertainty in measurement: a review of Monte Carlo simulation using Microsoft Excel for the calculation of uncertainties through functional relationships, including uncertainties in empirically derived constants. Clin Biochem Rev 2014;35:37-61.

14. Farrance I, Frenkel R, Badrick T. ISO/TS 20914:2019-a critical commentary. Clin Chem Lab Med 2020;58:1182-90.

15. Frenkel RB, Farrance I. Uncertainty in measurement: procedures for determining uncertainty with application to clinical laboratory calculations. Adv Clin Chem 2018;84:125-207. 